\title{
Training Through Distance Learning: An Assessment of Research Findings
}

\author{
Robert A. Wisher \\ U.S. Army Research Institute \\ Matthew V. Champagne, Jennifer L. Pawluk, and Angela Eaton \\ Rensselaer Polytechnic Institute \\ Consortium Research Fellows Program \\ David M. Thornton \\ George Mason University \\ Consortium Research Fellows Program \\ Christina K. Curnow \\ George Washington University \\ Consortium Research Fellows Program
}

\begin{abstract}
Advanced Training Methods Research Unit
Franklin L. Moses, Chief
\end{abstract}

U.S. Army Research Institute for the Behavioral and Social Sciences 5001 Eisenhower Avenue, Alexandria, Virginia 22333-5600

June 1999

Army Project Number 20363007A792

Personnel Performance and Training

Approved for public release; distribution is unlimited. 